\title{
Stark broadening of Sr I spectral lines ${ }^{\star}$
}

\author{
M.S. Dimitrijević ${ }^{1}$ and S. Sahal-Bréchot ${ }^{2}$ \\ 1 Astronomical Observatory, Volgina 7, 11050 Belgrade, Yugoslavia \\ 2 Laboratoire "Astrophysique, Atomes et Molécules", Département Atomes et Molécules en Astrophysique, Unité \\ associée au C.N.R.S. No. 812, Observatoire de Paris-Meudon, 92190 Meudon, France
}

Received March 13; accepted March 26, 1996

\begin{abstract}
Using a semiclassical approach, we have calculated electron-, proton-, and ionized helium-impact line widths and shifts for $33 \mathrm{Sr}$ I multiplets for perturber densities $10^{13} \mathrm{~cm}^{-3}$ (for stellar plasma research) and $10^{15}-$ $10^{18} \mathrm{~cm}^{-3}$ (for laboratory plasma research) and temperatures $T=2500-50000 \mathrm{~K}$.
\end{abstract}

Key words: lines: profile-atomic data

\section{Introduction}

Strontium lines are present in solar and stellar spectra. E.g. Komarov \& Basak (1993) have found neutral strontium lines in the spectra of Sun and two Praesepe's stars. They are also of interest since $\mathrm{Sr}$ is one of thermonuclear $\mathrm{s}$ - processes product in stars and its overabundance is observed in $\mathrm{CH}$ and metal deficient barium stars (Šleivyté \& Bartkevičius 1995). Neutral strontium lines are also of interest for the investigation of laboratory plasmas. Consequently, Kato et al. (1984) investigated wavelength shifts of Sr I lines emitted by an inductively coupled plasma and Karabut et al. (1980) dynamics of strontium line shapes during a pulsed discharge. Such lines have been considered theoretically as well by Davis (1972), for research of a laser - generated barium plasma. In order to continue our research of Stark broadening parameters needed for the investigation of astrophysical and laboratory plasmas and to provide the needed Stark broadening data, we have calculated within the semiclassical-perturbation formalism (Sahal-Bréchot 1969a,b) electron-, proton-, and ionized helium-impact line widths and shifts for $33 \mathrm{Sr}$ I multiplets. A summary of the formalism is given in Dimitrijević \& Sahal-Bréchot (1984).

\section{Results and discussion}

Energy levels for Sr I lines have been taken from Moore (1971). Oscillator strengths have been calculated by using

Send offprint requests to: M.S. Dimitrijević

${ }^{\star}$ Table 1 is only available in electronic form: The material published electronically can be accessed: by ftp at cdsarc.ustrasbg.fr or 130.79.128.5 or on WWW at: http//cdsweb.ustrasbg.fr/abstract.html the method of Bates \& Damgaard (1949) and the tables of Oertel \& Shomo (1968). For higher levels, the method described by Van Regemorter et al. (1979) has been used. We note that Gruzdev (1967) has found that the semiempirical and Hartree - Fock calculations of Sr I oscillator strengths agree fairly well with the $f$ values calculated by the method of Coulomb approximation.

In addition to electron-impact full halfwidths and shifts, Stark-broadening parameters due to proton-, and He II- impacts have been calculated. Our results for 33 Sr I multiplets are shown in Table 1 (accesible only in electronic form), for perturber densities $10^{13} \mathrm{~cm}^{-3}$ (for stellar plasma research) and $10^{15}-10^{18} \mathrm{~cm}^{-3}$ (for laboratory plasma research) and temperatures $T=2500-$ $50000 \mathrm{~K}$. We also specify a parameter $c$ (Dimitrijević \& Sahal-Bréchot 1984), which gives an estimate for the maximum perturber density for which the line may be treated as isolated when it is divided by the corresponding full width at half maximum. For each value given in Table 1, the collision volume $(V)$ multiplied by the perturber density $(N)$ is much less than one and the impact approximation is valid (Sahal-Bréchot 1969a,b). Values for $N V>0.5$ are not given and values for $0.1<N V \leq 0.5$ are denoted by an asterisk. When the impact approximation is not valid, the ion broadening contribution may be estimated by using quasistatic approach (Sahal-Bréchot 1991; Griem 1974). In the region between where neither of these two approximations is valid, a unified type theory should be used. For example in Barnard et al. (1974), a simple analytical formulas for such a case are given. The accuracy of the results obtained decreases when broadening by ion interactions becomes important. 
Acknowledgements. This work is a part of the project "Physics and dynamics of celestial bodies", supported by Ministry of Science and Technology of Serbia.

\section{References}

Barnard A.J., Cooper J., Smith E.W., 1974, JQSRT 14, 1025

Bates D.R., Damgaard A., 1949, Trans. Roy. Soc. London Ser. A 242, 101

Davis J., 1972, JQSRT 12, 1351

Dimitrijević M.S., Sahal-Bréchot S., 1984, JQSRT 31, 301

Griem H.R., 1974, Spectral Line Broadening by Plasmas. Academic Press, New York

Gruzdev P.F., 1967, Opt. Spektrosk. 22, 169

Karabut E.K., Kravchenko V.F., Mikhalevskii V.S., 1980, Opt.
Spectrosc. 48, 386

Kato K., Fukushima H., Nakajima T., 1984, Spectrochim. Acta B 39, 979

Komarov N.S., Basak N.Yu., 1993, Astron. Zh. 70, 111

Moore C.E., 1971, Atomic Energy Levels II, NSRDS-NBS 35, Washington

Oertel G.K., Shomo L.P., 1968, ApJS 16, 175

Sahal-Bréchot S., 1969a, A\&A 1, 91

Sahal-Bréchot S., 1969b, A\&A 2, 322

Sahal-Bréchot S., 1991, A\&A 245, 322

Šleivyté J., Bartkevičius A., 1995, Vilniaus Astron. Obs. Biuletenis 85, 3

Van Regemorter H., Hoang Binh Dy, Prud'homme M., 1979, J. Phys. B 12, 1073 